\title{
The Status of the Construction of MICE Step IV
}

\author{
Celeste Pidcott ${ }^{*}$ \\ University of Warwick \\ E-mail: c.e.pidcottewarwick.ac.uk
}

The International Muon Ionization Cooling Experiment will provide the demonstration of ionization cooling. The experiment is being built in a series of Steps. Step IV, which consists of a tracking spectrometer upstream and downstream of an absorber/focus-coil (AFC) module will be completed in 2015. In this configuration, the emittance of the muon beam upstream and downstream of the absorber will be measured precisely allowing the change in emittance and the factors that determine ionization cooling to be studied in detail. The absorber is a 22 litre volume of liquid hydrogen placed inside a superconducting focusing coil. The properties of lithium hydride, and possibly other absorber materials, will also be studied. All the components of Step IV have been manufactured and integration of the experiment in the MICE Hall at the Rutherford Appleton Laboratory is underway.

16th International Workshop on Neutrino Factories and Future Neutrino Beam Facilities 25 -30 August, 2014

University of Glasgow, United Kingdom

\footnotetext{
* Speaker.

${ }^{\dagger}$ On behalf of the MICE collaboration
} 


\section{Introduction}

MICE is a proof of principle experiment, designed to demonstrate the feasibility of ionization cooling as a method of reducing the emittance of muon beams, based at the Rutherford Appleton Laboratory (RAL), UK. Muon beams are proposed for use in future particle physics facilities, such as a Neutrino Factory or a Muon Collider. Due to the short muon lifetime, existing cooling techniques are unsuitable, and so a new technique is proposed - ionization cooling, whereby the muon beam is passed through a material (an absorber), reducing both the transverse and longitudinal momentum, and the beam is then re-accelerated to restore the longitudinal momentum.

In the Step IV configuration, MICE will study the material properties of $\mathrm{LH}_{2}$ and $\mathrm{LiH}$, and the change in the normalised transverse emittance of the beam. The Step IV configuration, with particle detectors included, is shown in figure 1. Tracking spectrometers (trackers) upstream and downstream of an absorber module measure the emittance of the beam before and after it passes through the absorber, and a diffuser placed before the upstream spectrometer can enlarge the emittance of the beam entering the channel.
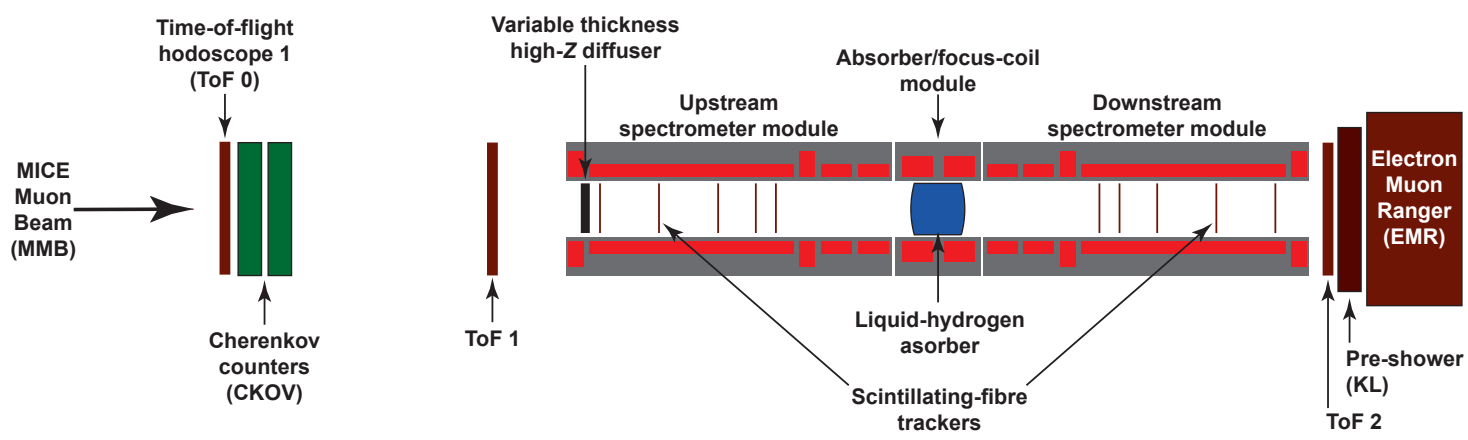

Figure 1: The Step IV configuration of MICE.

\section{Beamline}

The MICE Muon Beam, shown in figure 2, provides the muon beam for the experiment. The beam is produced by dipping a titanium target into the beam of the $800 \mathrm{MeV}$ ISIS proton synchrotron, generating pions which are then captured by a quadrupole triplet (Q1-3). After momentum selection in the first dipole magnet (D1), the pions are then transported into the 5T decay solenoid (DS), which increases the number of muons reaching the second dipole magnet (D2), where a second momentum selection occurs. The muons are then directed into two further quadrupole triplets, (Q4-6 and Q7-9), to be transported to the cooling channel [1]. The MICE Muon Beam has been constructed, and the beam characterisation, using time of flight (TOF), Cherenkov (CKOV), and KL detectors was completed in August 2010 [2].

\section{Particle Identification}

Particle Identification (PID) is required in MICE to reject pion contamination of the beam upstream of the cooling channel, and downstream of the channel to reject electrons/positrons pro- 


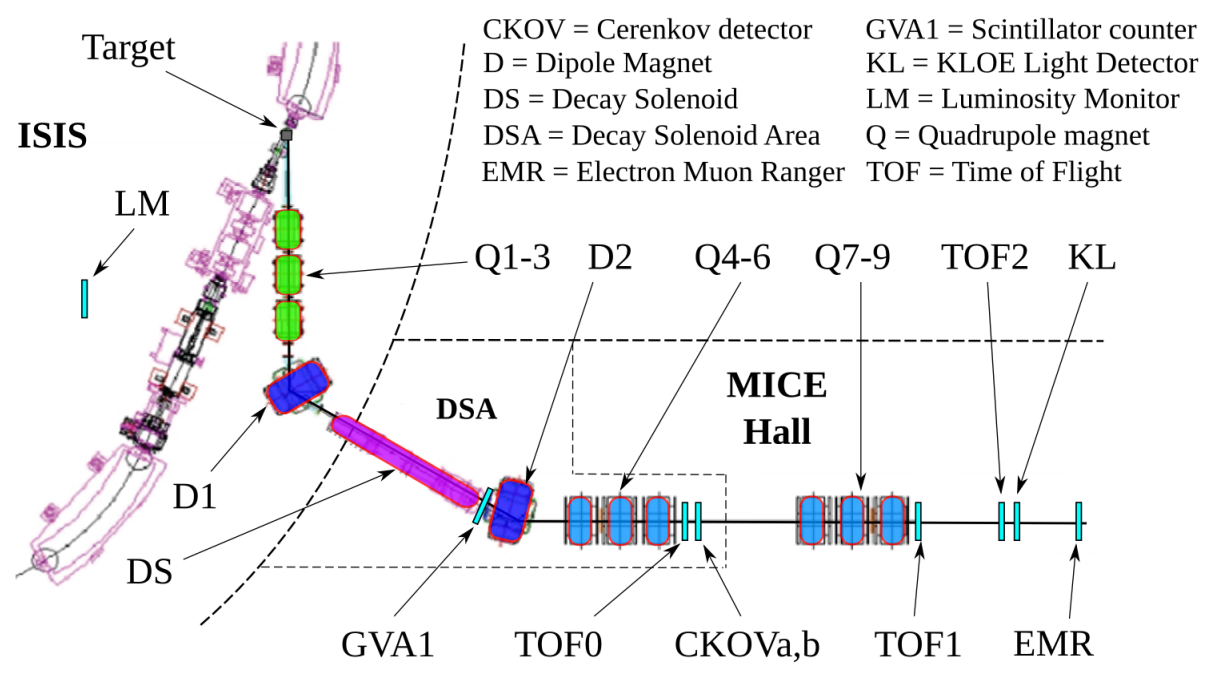

Figure 2: The MICE beamline and PID detectors, in the configuration used at Step I. At Step IV, the cooling channel will be between TOF1 and TOF2, and the downstream detectors (TOF2, KL, EMR) will be adjacent to each other.

duced from muon decays. The PID system in Step IV consists of two TOF detectors (TOF0 and TOF1) and two aerogel CKOV detectors upstream, and a third TOF detector (TOF2) and two calorimeters, the KLOE Light (KL) and Electron-Muon Ranger (EMR), downstream [3]. All of the PID detectors are installed in the MICE hall, and data was first taken with all detectors in October 2013.

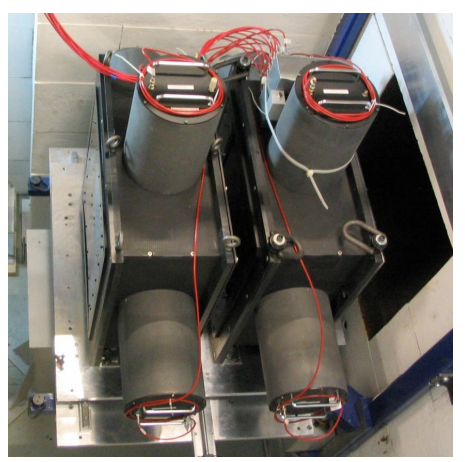

(a) Cherenkov detectors

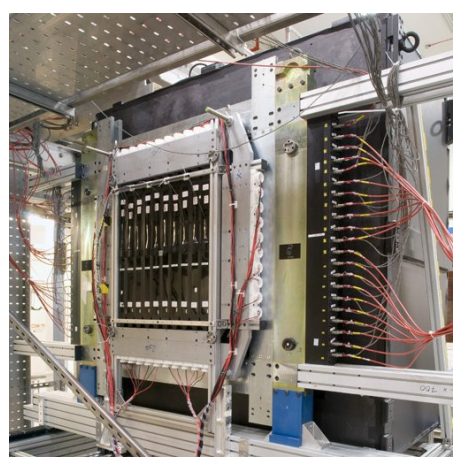

(b) TOF2 and KL detectors

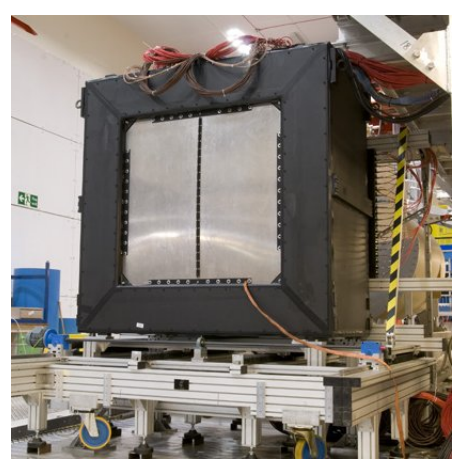

(c) EMR detector

Figure 3: PID detectors in MICE.

\section{Status of Step IV Components}

\subsection{Tracking Spectrometers and Spectrometer Solenoids}

The emittance measurements in MICE will be performed using two scintillating fibre trackers, installed within 4T spectrometer solenoids. Each tracker is composed of 5 stations, and each station is in turn composed of three planes of scintillating fibres [4]. The solenoids have been mapped, and 
both the upstream and downstream trackers have been fitted into the solenoids and installed in the MICE Hall, as can be seen in figure 4.

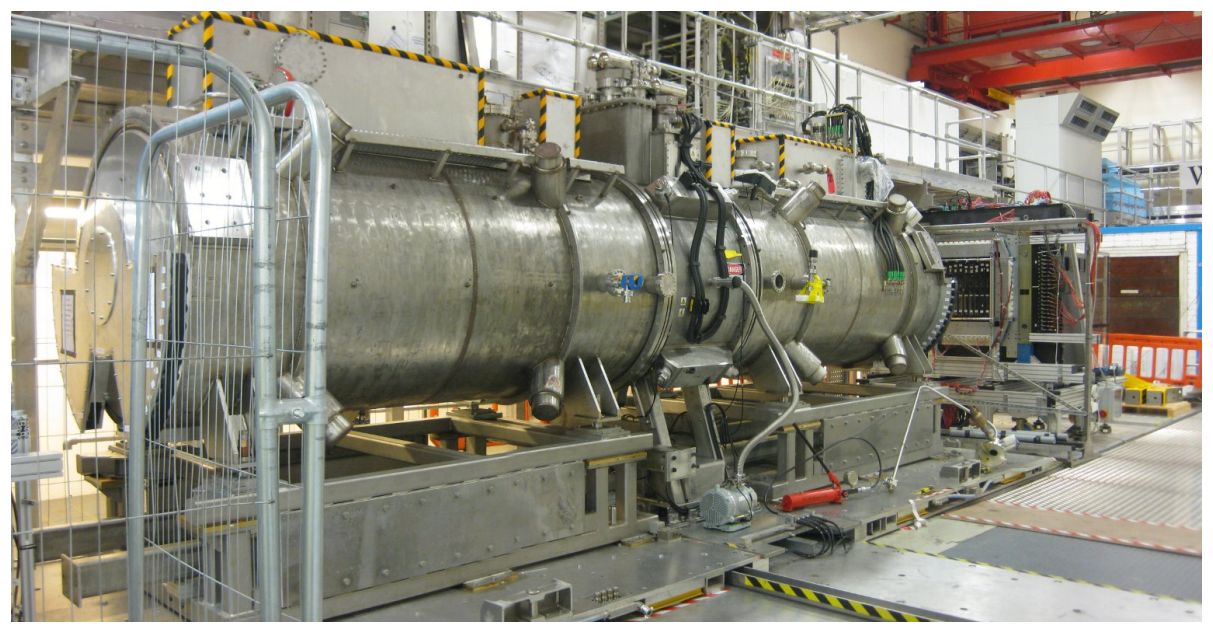

Figure 4: The spectrometer solenoids, with the trackers fitted, installed in the MICE hall. In between the two solenoids is the focus coil module.

\subsection{Absorbers and Focus Coil}

The focus coil (FC) module consists of two coils, which can operate in either flip mode, whereby the coils have opposite polarity, or solenoid (non-flip) mode, where the polarity of the coils is the same. Two FC modules have been manufactured, although only one will be required for Step IV.

The two absorbers to be studied in Step IV are liquid hydrogen $\left(\mathrm{LH}_{2}\right)$ and lithium-hydride ( $\mathrm{LiH})$. The $\mathrm{LH}_{2}$ will be contained within an aluminium vessel produced and tested by KEK. The vessel has two ultra thin aluminium windows, designed to minimise the amount of aluminium the beam passes through. The vessel is shown in figure $5 \mathrm{~b}$. It has been delivered to RAL and the $\mathrm{LH}_{2}$ delivery system has been tested. The solid $\mathrm{LiH}$ absorber, a $65 \mathrm{~mm}$ thick disk, shown in figure $5 \mathrm{c}$, and its support structure, have been made in the USA and delivered to RAL.

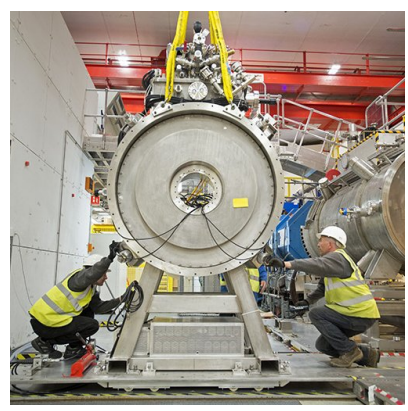

(a) Focus Coil

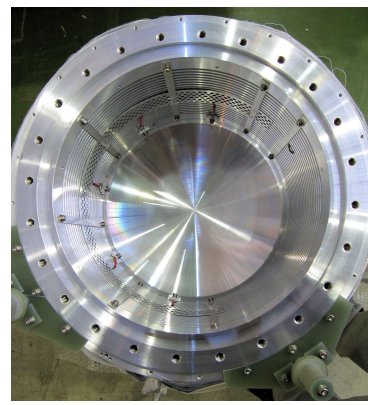

(b) $\mathrm{LH}_{2}$ vessel

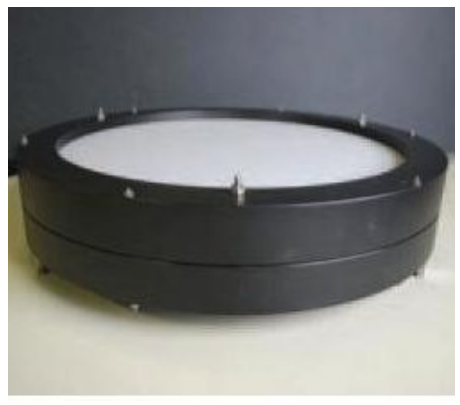

(c) $\mathrm{LiH}$ absorber

Figure 5: One of the MICE focus coil modules, and the absorbers to be studied in Step IV 


\subsection{Diffuser}

The diffuser is inserted into the upstream spectrometer solenoid, upstream of the tracker module. It consists of a stainless steel drum which contains four irises; two brass irises and two tungsten irises, all with different thicknesses, which can introduce up to $3 \mathrm{X}_{0}$ of material to the beam. The irises are pneumatically actuated, and can be operated remotely, with different combinations of open/closed irises producing a range of input emittances. The diffuser is now installed in the upstream spectrometer solenoid, as shown in figure 6.

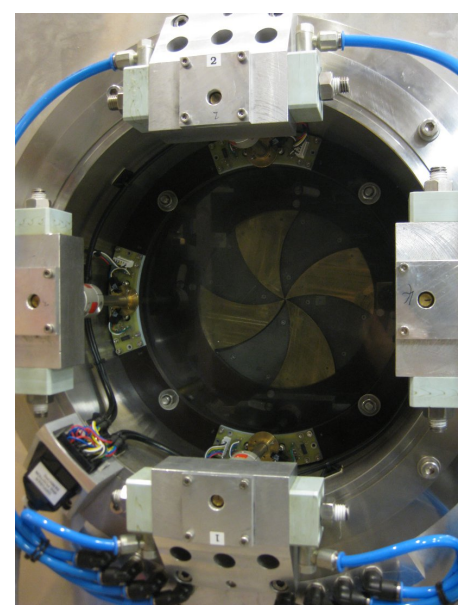

Figure 6: The diffuser installed within the upstream spectrometer solenoid.

\subsection{Partial Return Yoke}

A partial return yoke (PRY) will be installed to protect other equipment inside the MICE hall from the magnetic fields of the superconducting magnets. The PRY, which is shown in figure 7, reduces the 3-4 $\mathrm{T}$ on-axis fields to less than $1 \mathrm{mT}$ externally. Orders have been placed for the components of the PRY, and work is underway in the MICE hall on installation of the floor fixings and the legs of the PRY.

\section{Conclusions}

Construction of Step IV is at an advanced stage. The beamline, PID detectors, and the main apparatus of the cooling channel are all in place in the MICE hall, as can be seen in figure 8 . Whilst waiting for delivery and installation of the PRY, the data taking capabilities of the experiment will be tested, and calibration, alignment and precommissioning of the beamline will occur. Construction of Step IV will be completed, and commissioning begin, in the summer of 2015. 


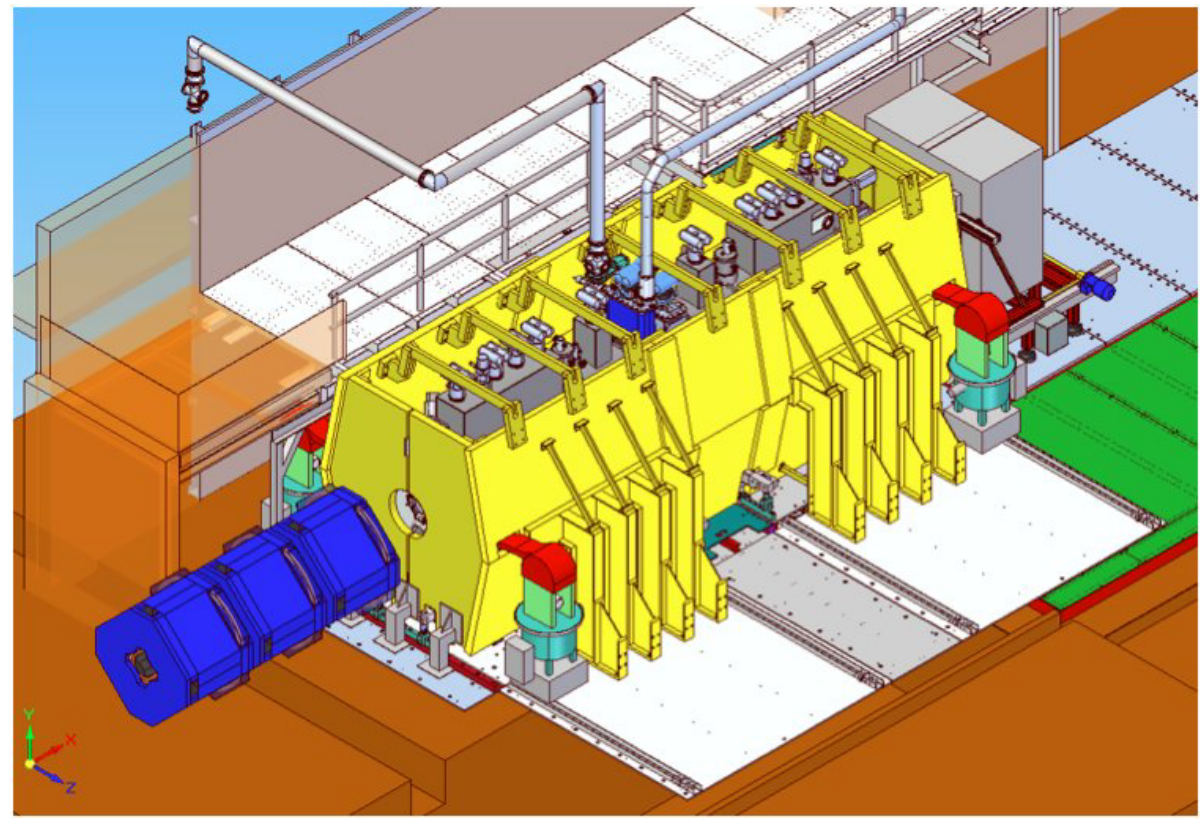

Figure 7: Schematic of the PRY around the Step IV cooling channel.

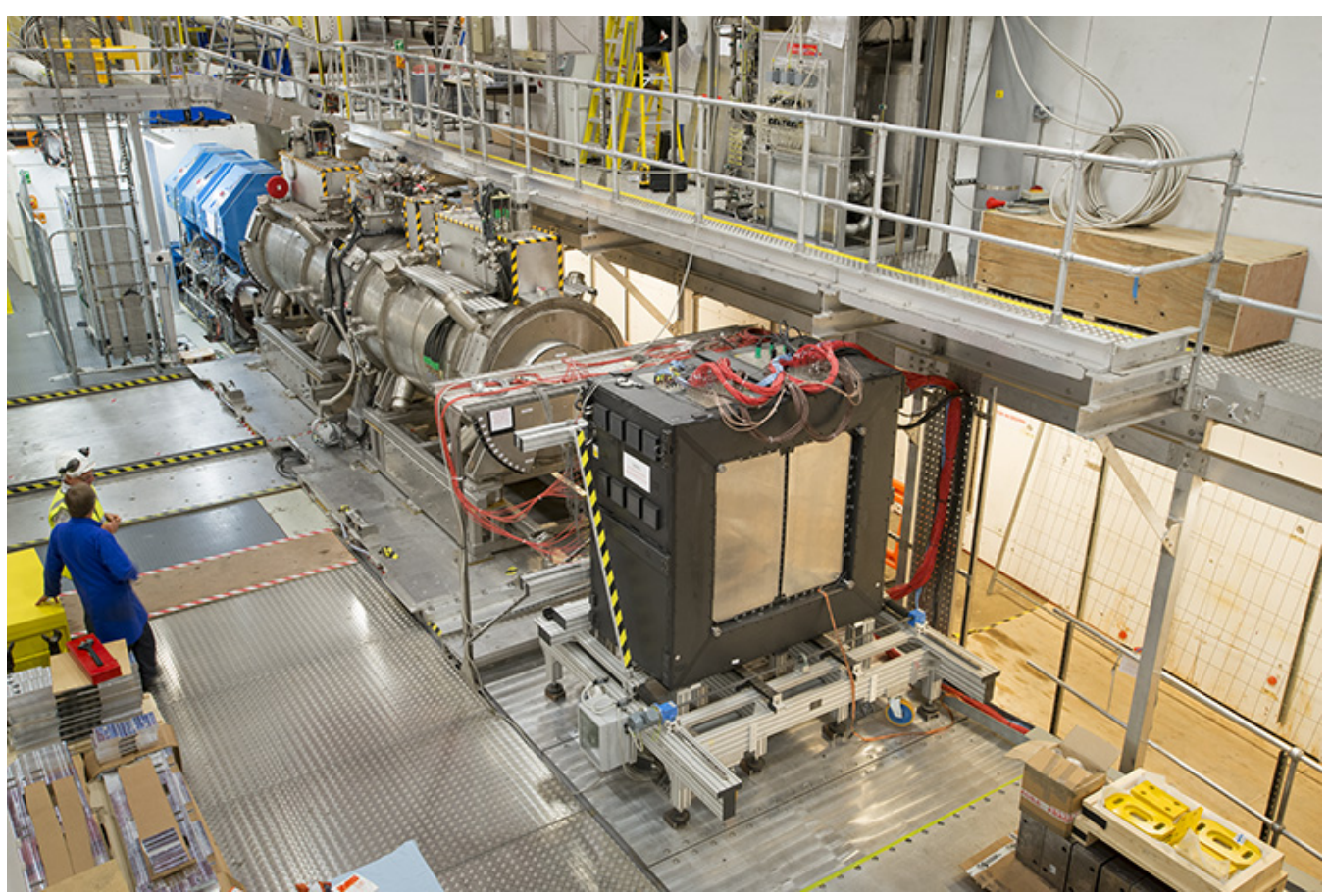

Figure 8: Photograph of the MICE hall in August 2014. The EMR can be seen in the foreground, immediately downstream of TOF2 and the KL (obscured from view). Upstream of these the spectrometer solenoids and focus coil can be seen, and upstream of them is the quadrupole triplet Q7-9 in blue. 


\section{References}

[1] M. Bogomilov, et al., The MICE Muon Beam on ISIS and the beam-line instrumentation of the Muon Ionization Cooling Experiment, JINST 7 (2012) P05009, arXiv: 1203.408 9v2.

[2] D. Adams, et al., Characterisation of the muon beams for the Muon Ionisation Cooling Experiment, Eur. Phys. J. C73 (2013) 2582, arXiv:1306.1509v2, 2013.

[3] D. A. Sanders, MICE Particle Identification Systems, in proceedings of PACO9 Conference, arXiv:0910.1332v1, 2009.

[4] M. Ellis, et al., The design, construction and performance of the MICE scintillating fibre trackers, arXiv:1005.3491v2, 2010. 